\title{
The MSSM without gluinos; an effective field theory for the stop sector
}

\author{
Jason Aebischer ${ }^{1, \mathrm{a}}$, Andreas Crivellin ${ }^{2, \mathrm{~b}}$, Christoph Greub ${ }^{1, \mathrm{c}}$, Youichi Yamada ${ }^{3, \mathrm{~d}}$ \\ ${ }^{1}$ Albert Einstein Center for Fundamental Physics, Institute for Theoretical Physics, University of Bern, 3012 Bern, Switzerland \\ 2 Paul Scherrer Institut (PSI), 5232 Villigen, Switzerland \\ ${ }^{3}$ Department of Physics, Tohoku University, Sendai 980-8578, Japan
}

Received: 20 June 2017 / Accepted: 17 October 2017 / Published online: 6 November 2017

(C) The Author(s) 2017. This article is an open access publication

\begin{abstract}
In this article we study the MSSM with stops and Higgs scalars much lighter than gluinos and squarks of the first two generations. In this setup, one should use an effective field theory with partial supersymmetry in which the gluino and heavy squarks are integrated out in order to connect SUSY parameters (given at a high scale) to observables in the stop sector. In the construction of this effective theory, valid below the gluino mass scale, we take into account $O\left(\alpha_{3}\right)$ and $O\left(Y_{t, b}^{2}\right)$ effects and calculate the matching as well as the renormalization group evolution. As a result, the running of the parameters for the stop sector is modified with respect to the full MSSM and SUSY relations between parameters are broken. We show that for some couplings sizable numerical differences exist between the effective field theory approach and the naive calculation based on the MSSM running.
\end{abstract}

\section{Introduction}

There are several theoretical arguments for a light stop in supersymmetric theories. Foremost, in natural supersymmetry (SUSY) light stops are required to cancel the quadratic divergence of the Higgs mass originating from the selfenergy involving a top quark, while the other supersymmetric partners can be much heavier $[1,2]$ due to the smaller couplings to the Higgs. Moreover, the renormalization group equations (RGE) of the minimal supersymmetric standard model (MSSM) generically drive the bilinear mass term parameters of the third generation squarks to lower values (compared to the first two generations) due to their nonnegligible Yukawa couplings [3-8].

\footnotetext{
a e-mail: aebischer@itp.unibe.ch

be-mail: andreas.crivellin@cern.ch

ce-mail: greub@itp.unibe.ch

de-mail: yamada@tuhep.phys.tohoku.ac.jp
}

Although the measured Higgs mass of around $125 \mathrm{GeV}$ [9, 10] prefers rather heavy (around the $\mathrm{TeV}$ scale) [11-13] rather than light stops in the MSSM, this is not necessarily the case in the NMSSM [14], in $\lambda$ SUSY models [15], models with light sneutrinos [16] or in supersymmertic models with additional D-term [17] or F-term [18] contributions to the scalar potential. Also large (or even maximal [19-21]) stop mixing angles help to get the right Higgs mass with rather light stops.

LHC searches for top squarks (using simplified models) set a lower bound on its mass of around $m_{\tilde{t}_{1}}=300 \mathrm{GeV}$, which however heavily depends on the neutralino mass. Depending on the stop and the neutralino mass, different decay modes are studied. For the decay channel $\tilde{t}_{1} \rightarrow t \tilde{\chi}_{1}^{0}$ [22-24], the limits are quite stringent, even though for light neutralinos very light stops cannot be excluded due to the high $t \bar{t}$-background [25]. The three-body decay $\tilde{t}_{1} \rightarrow W b \tilde{\chi}_{1}^{0}$ was analyzed theoretically in [26] and experimentally in [27]. Finally the decay $\tilde{t}_{1} \rightarrow c \tilde{\chi}_{1}^{0}$ and the less important four-body decay $\tilde{t}_{1} \rightarrow \tilde{\chi}_{1}^{0} d_{i} f \bar{f}^{\prime}$ are treated in [28-30] and constraints were derived by the ATLAS collaboration from the monojet analysis in [31]. Some bounds can be avoided in kinematic boundary regions or once non-minimal flavor violation is included. However, recently efforts of closing these gaps have been made [32-35] and stops should in general not be lighter than $300 \mathrm{GeV}$. Nevertheless, the mass bound for the stop is still weaker than the strong bounds on the squark masses of the first two generations and also on the gluino mass $[36,37]$. For sbottom quarks LHC searches suggest masses of above $800 \mathrm{GeV}[38,39]$. The bounds on sparticles with EW interactions only are much less stringent [40-44]. For example, in the case of heavy winos the Higgsino mass parameter $\mu$ has only to be larger than $350 \mathrm{GeV}$ [45]. It can be shown, however, that by changing the assumptions on the composition of charginos and neutralinos, collider limits can get even further weakened [46-49]. For the Higgs bosons, dif- 
ferent fits [50-58] suggest an alignment limit, in which the lightest CP-even Higgs boson takes the role of the SM Higgs. Collider limits on non-SM Higgs bosons for large values of $\tan \beta$ suggest that $\mathrm{CP}$-odd Higgs bosons should be heavier than $800 \mathrm{GeV}[59,60]$.

If the gluino (or the squarks of the first two generations $[2,61]$ ) is much heavier than the stops, an effective theory (EFT) with partial SUSY must be constructed in which the gluino (squarks) is integrated out [62-64]. Such a hierarchy can for example be achieved for MSSM-like models in a Scherk-Schwarz breaking scenario [65-68]. The construction of the effective theory for the stop sector is the goal of this article. Assuming a common large mass of order $M$ for the gluino and the squarks of the first two generations, we compute the matching condition between the full MSSM and the effective theory, including one-loop contributions which are enhanced by powers of $M$. Furthermore, since some supermultiplets are partially integrated out in the effective theory, the supersymmetric relations between gauge/Yukawa couplings, gaugino/Higgsino couplings and four-scalar couplings are broken in the effective theory by radiative corrections. Therefore, these couplings in the effective theory have an independent renormalization group evolution, as discussed in [62,69-76] mainly for the gauginomatter couplings.

This article is structured as follows: In the next section we establish our effective theory for the stop sector and calculate the matching as well as the running of the relevant parameters at order $\alpha_{3}=g_{3}^{2} /(4 \pi), Y_{t}^{2}$ and $Y_{b}^{2}$ (neglecting $O\left(g_{1}^{2}\right), O\left(g_{2}^{2}\right)$ and Higgs self-coupling effects). This section is followed by a numerical analysis in Sect. 3. Finally we conclude in Sect. 4.

\section{The effective theory for the stop sector}

The aim of this section is to construct the effective theory for the MSSM stop sector, including $O\left(\alpha_{3}, Y_{t, b}^{2}\right)$ and enhanced effects. As noted before, we assume that the gluino and the squarks of the first two generations are much heavier, with masses of the order $M$, than the stops, the Higgs scalars and the Higgsinos. The left-handed sbottom is also assumed to be light such that it remains in the effective theory, forming an $S U$ (2) multiplet with the left-handed stop. However, we assume that the right-handed sbottom is heavy, with the mass of the order $M$. Therefore, we consider the following effective Lagrangian which is valid below the scale $M$ :

$$
\begin{aligned}
\mathcal{L}_{\mathrm{eff}}= & \mathcal{L}_{K}-\bar{m}_{2}^{2} H_{u}^{\dagger} H_{u}-\bar{m}_{1}^{2} H_{d}^{\dagger} H_{d}-V\left(H_{u}, H_{d}\right) \\
& \left.+\bar{m}_{12}^{2} H_{d} \cdot H_{u}-\bar{\mu} \tilde{H}_{U} \cdot \tilde{H}_{D}+\text { (h.c. }\right) \\
& -\bar{m}_{\tilde{Q}}^{2} \tilde{q}_{L}^{\dagger} \tilde{q}_{L}-\bar{m}_{\tilde{t}}^{2} \tilde{t}_{R}^{\dagger} \tilde{t}_{R} \\
& \left.-\bar{Y}_{t} \bar{t}_{R} q_{3 L} \cdot H_{u}-\bar{Y}_{b} \bar{b}_{R} H_{d} \cdot q_{3 L}+\text { (h.c. }\right) \\
& -\lambda_{1}^{u}\left(\tilde{q}_{L}^{\dagger} \tilde{q}_{L}\right)\left(H_{u}^{\dagger} H_{u}\right)-\lambda_{2}^{u}\left(\tilde{q}_{L}^{\dagger} H_{u}\right)\left(H_{u}^{\dagger} \tilde{q}_{L}\right)
\end{aligned}
$$

$$
\begin{aligned}
& -\lambda_{3}^{u}\left(\tilde{t}_{R}^{\dagger} \tilde{t}_{R}\right)\left(H_{u}^{\dagger} H_{u}\right) \\
& -\lambda_{1}^{d}\left(\tilde{q}_{L}^{\dagger} \tilde{q}_{L}\right)\left(H_{d}^{\dagger} H_{d}\right)-\lambda_{2}^{d}\left(\tilde{q}_{L}^{\dagger} H_{d}\right)\left(H_{d}^{\dagger} \tilde{q}_{L}\right) \\
& -\lambda_{3}^{d}\left(\tilde{t}_{R}^{\dagger} \tilde{t}_{R}\right)\left(H_{d}^{\dagger} H_{d}\right) \\
& -\lambda_{4}\left(\tilde{q}_{L i}^{\dagger} \tilde{q}_{L i}\right)\left(\tilde{q}_{L j}^{\dagger} \tilde{q}_{L j}\right)-\lambda_{5}\left(\tilde{q}_{L i}^{\dagger} \tilde{q}_{L j}\right)\left(\tilde{q}_{L j}^{\dagger} \tilde{q}_{L i}\right) \\
& -\lambda_{6}\left(\tilde{q}_{L i}^{\dagger} \tilde{q}_{L i}\right)\left(\tilde{t}_{R}^{\dagger} \tilde{t}_{R}\right)-\lambda_{7}\left(\tilde{q}_{L i}^{\dagger} \tilde{t}_{R}\right)\left(\tilde{t}_{R}^{\dagger} \tilde{q}_{L i}\right) \\
& -\lambda_{8}\left(\tilde{t}_{R}^{\dagger} \tilde{t}_{R}\right)\left(\tilde{t}_{R}^{\dagger} \tilde{t}_{R}\right) \\
& -\bar{A}_{t} \tilde{t}_{R}^{\dagger} \tilde{q}_{L} \cdot H_{u}+\bar{\mu}_{t} \tilde{t}_{R}^{\dagger} H_{d}^{\dagger} \tilde{q}_{L}+(\text { h.c. }) \\
& -\bar{Y}_{q_{3 L}} \tilde{t}_{R}^{\dagger} q_{3 L} \cdot \tilde{H}_{U}-\bar{Y}_{t_{R}} \bar{t}_{R} \tilde{q}_{L} \cdot \tilde{H}_{U} \\
& -\bar{Y}_{b_{R}} \bar{b}_{R} \tilde{H}_{D} \cdot \tilde{q}_{L}+(\text { h.c. }),
\end{aligned}
$$

with partial supersymmetry. Here $\mathcal{L}_{K}$ denotes the kinetic terms and gauge interactions, and $V\left(H_{u}, H_{d}\right)$ denotes the quartic couplings of the Higgs doublets $\left(H_{u}, H_{d}\right)$. For the interactions involving four squarks, the $S U$ (3) color indices are contracted within the parentheses. Similarly, the $S U$ (2) indices in the two-squark-two-Higgs interactions are contracted within the parentheses. $i, j$ are the $S U(2)$ indices and the dot denotes the contraction of $S U(2)$ indices as $A \cdot B=A_{1} B_{2}-A_{2} B_{1}$. For simplicity, we also assume that the electroweak gauginos and sleptons are heavy. However, since we neglect $O\left(g_{1}^{2}\right), O\left(g_{2}^{2}\right)$ effects in the following, relaxing this assumption would leave our RGEs unchanged. We also ignore the non-holomorphic Higgs-quark couplings $\bar{t}_{R} H_{d}^{\dagger} q_{3 L}$ and $\bar{b}_{R} H_{u}^{\dagger} q_{3 L}$, which are induced at the loop level [77-84].

\subsection{Tree-level matching}

At the matching scale $M$ the Lagrangian of Eq. (1) has to be compared to the one of the full MSSM (see for example [8588]) which originates from the superpotential

$$
W=Y_{t} T^{c} Q \cdot H_{u}+Y_{b} B^{c} H_{d} \cdot Q+\mu H_{u} \cdot H_{d},
$$

the soft SUSY breaking terms

$$
\begin{aligned}
V_{\text {soft }}= & m_{\tilde{Q}}^{2} \tilde{q}_{L}^{\dagger} \tilde{q}_{L}+m_{\tilde{t}}^{2} \tilde{t}_{R}^{\dagger} \tilde{t}_{R}+m_{H_{d}}^{2} H_{d}^{\dagger} H_{d} \\
& +m_{H_{u}}^{2} H_{u}^{\dagger} H_{u}+m_{\tilde{b}_{R}}^{2} \tilde{b}_{R}^{\dagger} \tilde{b}_{R} \\
& +A_{t} \tilde{t}_{R}^{\dagger} \tilde{q}_{L} \cdot H_{u}+A_{b} \tilde{b}_{R}^{\dagger} H_{d} \cdot \tilde{q}_{L} \\
& -m_{H_{d} H_{u}}^{2} H_{d} \cdot H_{u}+\text { (h.c.) },
\end{aligned}
$$

and the $D$ terms

$V_{D}=\frac{g_{3}^{2}}{2}\left(\tilde{q}_{L}^{\dagger} T^{A} \tilde{q}_{L}-\tilde{t}_{R}^{\dagger} T^{A} \tilde{t}_{R}-\tilde{b}_{R}^{\dagger} T^{A} \tilde{b}_{R}\right)^{2}$,

where $T^{A}$ are the generators of $S U(3)$ in the fundamental representation.

The matching conditions for the bilinear terms and the trilinear couplings are

$$
\bar{Y}_{t}=Y_{t}, \quad \bar{Y}_{b}=Y_{b}, \quad \bar{Y}_{q_{3 L}}=Y_{t},
$$




$$
\begin{aligned}
\bar{Y}_{t_{R}} & =Y_{t}, \quad \bar{Y}_{b_{R}}=Y_{b}, \quad \bar{A}_{t}=A_{t}, \\
\bar{\mu} & =\mu, \quad \bar{\mu}_{t}=\mu Y_{t}, \quad \bar{m}_{2}^{2}=m_{H_{u}}^{2}+\mu^{2}, \\
\bar{m}_{1}^{2} & =m_{H_{d}}^{2}+\mu^{2}, \\
\bar{m}_{12}^{2} & =m_{H_{d} H_{u}}^{2}, \quad \bar{m}_{\tilde{Q}}^{2}=m_{\tilde{Q}}^{2}, \quad \bar{m}_{\tilde{t}}^{2}=m_{\tilde{t}}^{2} .
\end{aligned}
$$

The couplings between squarks and Higgs bosons are generated by F- and D-terms in the MSSM Lagrangian. At the scale $M$, they are given by

$\lambda_{1}^{u}=Y_{t}^{2}, \quad \lambda_{2}^{u}=-Y_{t}^{2}, \quad \lambda_{3}^{u}=Y_{t}^{2}$,

$\lambda_{1}^{d}=Y_{b}^{2}, \quad \lambda_{2}^{d}=-Y_{b}^{2}, \quad \lambda_{3}^{d}=0$,

$\lambda_{4}=-\frac{1}{12} g_{3}^{2}, \quad \lambda_{5}=\frac{1}{4} g_{3}^{2}, \quad \lambda_{6}=\frac{1}{6} g_{3}^{2}$,

$\lambda_{7}=-\frac{1}{2} g_{3}^{2}+Y_{t}^{2}, \quad \lambda_{8}=\frac{1}{6} g_{3}^{2}$,

keeping only Yukawa couplings and $g_{3}$.

\subsection{One-loop matching}

For the matching, we need to include the one-loop effects enhanced by powers of $M$ since their contributions may be comparable to the tree level ones shown in the previous subsection. They can only appear in bilinear and trilinear terms, as seen by dimensional analysis. The bilinear terms receive the following shifts at the matching scale $\mu_{R}=M$ :

$$
\begin{aligned}
\Delta \bar{m}_{2}^{2}= & 0 \\
\Delta \bar{m}_{1}^{2}= & -\frac{3}{16 \pi^{2}}\left(Y_{b}^{2} m_{\tilde{b}_{R}}^{2}+A_{b}^{2}\right)\left(1-\log \left(\frac{m_{\tilde{b}_{R}}^{2}}{M^{2}}\right)\right), \\
\Delta \bar{m}_{\tilde{Q}}^{2}= & -\frac{1}{16 \pi^{2}}\left(Y_{b}^{2} m_{\tilde{b}_{R}}^{2}+A_{b}^{2}\right)\left(1-\log \left(\frac{m_{\tilde{b}_{R}}^{2}}{M^{2}}\right)\right) \\
& +\frac{\alpha_{3} C_{F}}{\pi} m_{\tilde{g}}^{2}\left(1-\log \left(\frac{m_{\tilde{g}}^{2}}{M^{2}}\right)\right) \\
\Delta \bar{m}_{\tilde{t}}^{2}= & \frac{\alpha_{3} C_{F}}{\pi} m_{\tilde{g}}^{2}\left(1-\log \left(\frac{m_{\tilde{g}}^{2}}{M^{2}}\right)\right) \\
\Delta \bar{m}_{12}^{2}= & -\frac{3 A_{b} \mu Y_{b}}{16 \pi^{2}}\left(1-\log \left(\frac{m_{\tilde{b}_{R}}^{2}}{M^{2}}\right)\right) \\
\Delta \bar{\mu}= & 0 .
\end{aligned}
$$

For the trilinear term the shift reads

$$
\begin{aligned}
\Delta \bar{A}_{t}= & -\frac{A_{b} Y_{t} Y_{b}}{16 \pi^{2}}\left(1-\log \left(\frac{m_{\tilde{b}_{R}}^{2}}{M^{2}}\right)\right) \\
& -\frac{\alpha_{3} C_{F}}{\pi} m_{\tilde{g}} Y_{t}\left(1-\log \left(\frac{m_{\tilde{g}}^{2}}{M^{2}}\right)\right),
\end{aligned}
$$

$\Delta \bar{\mu}_{t}=0$.
All the other parameters relevant for the stop sector are dimensionless and therefore do not receive any $M$ enhanced corrections.

\subsection{Renormalization group evolution}

The running of the full MSSM parameters [3-7] is known at the two-loop level $[8,89-92]$. Here we give the one-loop beta functions to $\mathcal{O}\left(\alpha_{3}, Y_{t, b}^{2}\right)$ for the parameters of our effective theory in Eq. (1). The corresponding results for the full MSSM are summarized in the appendix. For the strong coupling constant we have $\left(t \equiv \log \mu_{R}\right.$, where $\mu_{R}$ denotes the renormalization scale)

$16 \pi^{2} \frac{\mathrm{d}}{\mathrm{d} t} \bar{g}_{3}=\left(-7+\frac{1}{2}\right) \bar{g}_{3}^{3}$,

where the first term on the right hand side is the SM contribution. The effective quark-quark-Higgs Yukawa couplings evolve according to

$$
\begin{aligned}
& 16 \pi^{2} \frac{\mathrm{d}}{\mathrm{d} t} \bar{Y}_{t}=\bar{Y}_{t}\left[-8 \bar{g}_{3}^{2}+\frac{9}{2} \bar{Y}_{t}^{2}+\frac{1}{2} \bar{Y}_{b}^{2}+\bar{Y}_{t_{R}}^{2}+\frac{1}{2} \bar{Y}_{q_{3 L}}^{2}\right], \\
& 16 \pi^{2} \frac{\mathrm{d}}{\mathrm{d} t} \bar{Y}_{b}=\bar{Y}_{b}\left[-8 \bar{g}_{3}^{2}+\frac{1}{2} \bar{Y}_{t}^{2}+\frac{9}{2} \bar{Y}_{b}^{2}+\bar{Y}_{b_{R}}^{2}+\frac{1}{2} \bar{Y}_{q_{3 L}}^{2}\right],
\end{aligned}
$$

while the evolution of the ones entering the Higgsino-quarksquark vertex is determined by

$$
\begin{aligned}
& 16 \pi^{2} \frac{\mathrm{d}}{\mathrm{d} t} \bar{Y}_{q_{3 L}} \\
& =\bar{Y}_{q_{3 L}}\left[-4 \bar{g}_{3}^{2}+\frac{1}{2} \bar{Y}_{t}^{2}+\frac{1}{2} \bar{Y}_{b}^{2}+4 \bar{Y}_{q_{3 L}}^{2}+\frac{3}{2} \bar{Y}_{t_{R}}^{2}\right] \\
& 16 \pi^{2} \frac{\mathrm{d}}{\mathrm{d} t} \bar{Y}_{t_{R}} \\
& =\bar{Y}_{t_{R}}\left[-4 \bar{g}_{3}^{2}+\bar{Y}_{t}^{2}+\frac{3}{2} \bar{Y}_{q_{3 L}}^{2}+\frac{7}{2} \bar{Y}_{t_{R}}^{2}+\bar{Y}_{b_{R}}^{2}\right] \\
& 16 \pi^{2} \frac{\mathrm{d}}{\mathrm{d} t} \bar{Y}_{b_{R}}=\bar{Y}_{b_{R}}\left[-4 \bar{g}_{3}^{2}+\bar{Y}_{b}^{2}+\bar{Y}_{t_{R}}^{2}+\frac{7}{2} \bar{Y}_{b_{R}}^{2}\right]
\end{aligned}
$$

For the Higgs mass parameters we find

$$
\begin{aligned}
16 \pi^{2} \frac{\mathrm{d}}{\mathrm{d} t} \bar{m}_{2}^{2}= & 6 \bar{Y}_{t}^{2} \bar{m}_{2}^{2}+6\left(2 \lambda_{1}^{u}+\lambda_{2}^{u}\right) \bar{m}_{\tilde{Q}}^{2} \\
& +6 \lambda_{3}^{u} \bar{m}_{\tilde{t}}^{2}+6 \bar{A}_{t}^{2}, \\
16 \pi^{2} \frac{\mathrm{d}}{\mathrm{d} t} \bar{m}_{1}^{2}= & 6 \bar{Y}_{b}^{2} \bar{m}_{1}^{2}+6\left(2 \lambda_{1}^{d}+\lambda_{2}^{d}\right) \bar{m}_{\tilde{Q}}^{2} \\
& +6 \lambda_{3}^{d} \bar{m}_{\tilde{t}}^{2}+6 \bar{\mu}_{t}^{2}, \\
16 \pi^{2} \frac{\mathrm{d}}{\mathrm{d} t} \bar{m}_{12}^{2}= & 3\left(\bar{Y}_{t}^{2}+\bar{Y}_{b}^{2}\right) \bar{m}_{12}^{2}+6 \bar{\mu}_{t} \bar{A}_{t},
\end{aligned}
$$


and for the bilinear squark mass terms

$$
\begin{aligned}
16 \pi^{2} \frac{\mathrm{d}}{\mathrm{d} t} \bar{m}_{\tilde{Q}}^{2}= & {\left[-8 \bar{g}_{3}^{2}+2 \bar{Y}_{t_{R}}^{2}+2 \bar{Y}_{b_{R}}^{2}+28 \lambda_{4}+20 \lambda_{5}\right] \bar{m}_{\tilde{Q}}^{2} } \\
& +\left(6 \lambda_{6}+2 \lambda_{7}\right) \bar{m}_{\tilde{t}}^{2} \\
& +\left(4 \lambda_{1}^{u}+2 \lambda_{2}^{u}\right) \bar{m}_{2}^{2}+\left(4 \lambda_{1}^{d}+2 \lambda_{2}^{d}\right) \bar{m}_{1}^{2} \\
& +2\left(\bar{A}_{t}^{2}+\bar{\mu}_{t}^{2}\right)-4\left(\bar{Y}_{t_{R}}^{2}+\bar{Y}_{b_{R}}^{2}\right) \bar{\mu}^{2} \\
16 \pi^{2} \frac{\mathrm{d}}{\mathrm{d} t} \bar{m}_{\tilde{t}}^{2}= & {\left[-8 \bar{g}_{3}^{2}+4 \bar{Y}_{q_{3 L}}^{2}+16 \lambda_{8}\right] \bar{m}_{\tilde{t}}^{2} } \\
& +\left(12 \lambda_{6}+4 \lambda_{7}\right) \bar{m}_{\tilde{Q}}^{2} \\
& +4 \lambda_{3}^{u} \bar{m}_{2}+4 \lambda_{3}^{d} \bar{m}_{1}+4\left(\bar{A}_{t}^{2}+\bar{\mu}_{t}^{2}\right) \\
& -8 \bar{Y}_{q_{3 L}}^{2} \bar{\mu}^{2} .
\end{aligned}
$$

The Higgsino mass in the effective theory evolves as

$16 \pi^{2} \frac{\mathrm{d}}{\mathrm{d} t} \bar{\mu}=\frac{3}{2}\left(\bar{Y}_{q_{3 L}}^{2}+\bar{Y}_{t_{R}}^{2}+\bar{Y}_{b_{R}}^{2}\right) \bar{\mu}$,

and the effective trilinear $H \tilde{q} \tilde{q}$ coupling as

$$
\begin{aligned}
16 \pi^{2} \frac{\mathrm{d}}{\mathrm{d} t} \bar{A}_{t}= & \bar{A}_{t}\left[-8 \bar{g}_{3}^{2}+2 \bar{Y}_{q_{3 L}}^{2}+\bar{Y}_{t_{R}}^{2}+\bar{Y}_{b_{R}}^{2}+3 \bar{Y}_{t}^{2}\right. \\
& \left.+2 \lambda_{1}^{u}-2 \lambda_{2}^{u}+2 \lambda_{3}^{u}+2 \lambda_{6}+6 \lambda_{7}\right], \\
16 \pi^{2} \frac{\mathrm{d}}{\mathrm{d} t} \bar{\mu}_{t}= & \bar{\mu}_{t}\left[-8 \bar{g}_{3}^{2}+2 \bar{Y}_{q_{3 L}}^{2}+\bar{Y}_{t_{R}}^{2}+\bar{Y}_{b_{R}}^{2}+3 \bar{Y}_{b}^{2}\right. \\
& \left.+2 \lambda_{1}^{d}+4 \lambda_{2}^{d}+2 \lambda_{3}^{d}+2 \lambda_{6}+6 \lambda_{7}\right] \\
& +4 \bar{Y}_{q_{3 L}} \bar{Y}_{b_{R}} \bar{Y}_{b} \bar{\mu} .
\end{aligned}
$$

Finally for the quartic $H H \tilde{q} \tilde{q}$ and $\tilde{q} \tilde{q} \tilde{q} \tilde{q}$ couplings one obtains

$$
\begin{aligned}
16 \pi^{2} \frac{\mathrm{d}}{\mathrm{d} t} \lambda_{1}^{u}= & 4\left(\lambda_{1}^{u}\right)^{2}+2\left(\lambda_{2}^{u}\right)^{2}+28 \lambda_{1}^{u} \lambda_{4}+20 \lambda_{1}^{u} \lambda_{5} \\
& +12 \lambda_{2}^{u} \lambda_{4}+4 \lambda_{2}^{u} \lambda_{5}+6 \lambda_{3}^{u} \lambda_{6} \\
& +2 \lambda_{3}^{u} \lambda_{7}+\left(-8 \bar{g}_{3}^{2}+6 \bar{Y}_{t}^{2}+2 \bar{Y}_{t_{R}}^{2}+2 \bar{Y}_{b_{R}}^{2}\right) \lambda_{1}^{u} \\
& -4 \bar{Y}_{t_{R}}^{2} \bar{Y}_{t}^{2},
\end{aligned}
$$

$16 \pi^{2} \frac{\mathrm{d}}{\mathrm{d} t} \lambda_{2}^{u}=8 \lambda_{1}^{u} \lambda_{2}^{u}+4\left(\lambda_{2}^{u}\right)^{2}+4 \lambda_{2}^{u} \lambda_{4}+12 \lambda_{2}^{u} \lambda_{5}$

$$
+\left(-8 \bar{g}_{3}^{2}+6 \bar{Y}_{t}^{2}+2 \bar{Y}_{t_{R}}^{2}+2 \bar{Y}_{b_{R}}^{2}\right) \lambda_{2}^{u},
$$

$16 \pi^{2} \frac{\mathrm{d}}{\mathrm{d} t} \lambda_{3}^{u}=12 \lambda_{1}^{u} \lambda_{6}+6 \lambda_{2}^{u} \lambda_{6}+4 \lambda_{1}^{u} \lambda_{7}+2 \lambda_{2}^{u} \lambda_{7}$

$$
\begin{aligned}
& +4\left(\lambda_{3}^{u}\right)^{2}+16 \lambda_{3}^{u} \lambda_{8} \\
& +\left(-8 \bar{g}_{3}^{2}+6 \bar{Y}_{t}^{2}+4 \bar{Y}_{q_{3 L}}^{2}\right) \lambda_{3}^{u}-4 \bar{Y}_{q_{3 L}}^{2} \bar{Y}_{t}^{2},
\end{aligned}
$$

$$
\begin{aligned}
16 \pi^{2} \frac{\mathrm{d}}{\mathrm{d} t} \lambda_{1}^{d}= & 4\left(\lambda_{1}^{d}\right)^{2}+2\left(\lambda_{2}^{d}\right)^{2}+28 \lambda_{1}^{d} \lambda_{4}+20 \lambda_{1}^{d} \lambda_{5} \\
& +12 \lambda_{2}^{d} \lambda_{4}+4 \lambda_{2}^{d} \lambda_{5}+6 \lambda_{3}^{d} \lambda_{6}+2 \lambda_{3}^{d} \lambda_{7} \\
& +\left(-8 \bar{g}_{3}^{2}+6 \bar{Y}_{b}^{2}+2 \bar{Y}_{t_{R}}^{2}+2 \bar{Y}_{b_{R}}^{2}\right) \lambda_{1}^{d} \\
& -4 \bar{Y}_{b_{R}}^{2} \bar{Y}_{b}^{2},
\end{aligned}
$$

$$
\begin{aligned}
16 \pi^{2} \frac{\mathrm{d}}{\mathrm{d} t} \lambda_{2}^{d}= & 8 \lambda_{1}^{d} \lambda_{2}^{d}+4\left(\lambda_{2}^{d}\right)^{2}+4 \lambda_{2}^{d} \lambda_{4}+12 \lambda_{2}^{d} \lambda_{5} \\
& +\left(-8 \bar{g}_{3}^{2}+6 \bar{Y}_{b}^{2}+2 \bar{Y}_{t_{R}}^{2}+2 \bar{Y}_{b_{R}}^{2}\right) \lambda_{2}^{d},
\end{aligned}
$$

$$
\begin{aligned}
16 \pi^{2} \frac{\mathrm{d}}{\mathrm{d} t} \lambda_{3}^{d}= & 12 \lambda_{1}^{d} \lambda_{6}+6 \lambda_{2}^{d} \lambda_{6}+4 \lambda_{1}^{d} \lambda_{7}+2 \lambda_{2}^{d} \lambda_{7} \\
& +4\left(\lambda_{3}^{d}\right)^{2}+16 \lambda_{3}^{d} \lambda_{8} \\
& +\left(-8 \bar{g}_{3}^{2}+6 \bar{Y}_{b}^{2}+4 \bar{Y}_{q_{3 L}}^{2}\right) \lambda_{3}^{d}-4 \bar{Y}_{q_{3 L}}^{2} \bar{Y}_{b}^{2},
\end{aligned}
$$

$$
\begin{aligned}
16 \pi^{2} \frac{\mathrm{d}}{\mathrm{d} t} \lambda_{4}= & 2\left(\lambda_{1}^{u}\right)^{2}+2 \lambda_{1}^{u} \lambda_{2}^{u}+2\left(\lambda_{1}^{d}\right)^{2}+2 \lambda_{1}^{d} \lambda_{2}^{d} \\
& +40 \lambda_{4}^{2}+40 \lambda_{4} \lambda_{5}+12 \lambda_{5}^{2}+3 \lambda_{6}^{2} \\
& +2 \lambda_{6} \lambda_{7}+\left(-16 \bar{g}_{3}^{2}+4\left(\bar{Y}_{t_{R}}^{2}+\bar{Y}_{b_{R}}^{2}\right)\right) \lambda_{4} \\
& +\frac{11}{12} \bar{g}_{3}^{4},
\end{aligned}
$$$$
16 \pi^{2} \frac{\mathrm{d}}{\mathrm{d} t} \lambda_{5}=\left(\lambda_{2}^{u}\right)^{2}+\left(\lambda_{2}^{d}\right)^{2}+24 \lambda_{4} \lambda_{5}+20 \lambda_{5}^{2}+\lambda_{7}^{2}
$$$$
+\left(-16 \bar{g}_{3}^{2}+4\left(\bar{Y}_{t_{R}}^{2}+\bar{Y}_{b_{R}}^{2}\right)\right) \lambda_{5}
$$$$
-2\left(\bar{Y}_{t_{R}}^{4}+\bar{Y}_{b_{R}}^{4}\right)+\frac{5}{4} \bar{g}_{3}^{4} \text {, }
$$

$$
\begin{aligned}
16 \pi^{2} \frac{\mathrm{d}}{\mathrm{d} t} \lambda_{6}= & \left(4 \lambda_{1}^{u}+2 \lambda_{2}^{u}\right) \lambda_{3}^{u}+\left(4 \lambda_{1}^{d}+2 \lambda_{2}^{d}\right) \lambda_{3}^{d}+28 \lambda_{4} \lambda_{6} \\
& +8 \lambda_{4} \lambda_{7}+20 \lambda_{5} \lambda_{6}+4 \lambda_{5} \lambda_{7} \\
& +4 \lambda_{6}^{2}+2 \lambda_{7}^{2}+16 \lambda_{6} \lambda_{8}+4 \lambda_{7} \lambda_{8} \\
& +\left(-16 \bar{g}_{3}^{2}+2 \bar{Y}_{t_{R}}^{2}+2 \bar{Y}_{b_{R}}^{2}+4 \bar{Y}_{q_{3 L}}^{2}\right) \lambda_{6} \\
& -4 \bar{Y}_{t_{R}}^{2} \bar{Y}_{q_{3 L}}^{2}+\frac{11}{6} \bar{g}_{3}^{4}
\end{aligned}
$$

$$
\begin{aligned}
16 \pi^{2} \frac{\mathrm{d}}{\mathrm{d} t} \lambda_{7}= & 4 \lambda_{4} \lambda_{7}+8 \lambda_{5} \lambda_{7}+8 \lambda_{6} \lambda_{7}+6 \lambda_{7}^{2}+4 \lambda_{7} \lambda_{8} \\
& +\left(-16 \bar{g}_{3}^{2}+2 \bar{Y}_{t_{R}}^{2}+2 \bar{Y}_{b_{R}}^{2}+4 \bar{Y}_{q_{3 L}}^{2}\right) \lambda_{7}+\frac{5}{2} \bar{g}_{3}^{4},
\end{aligned}
$$

$$
\begin{aligned}
16 \pi^{2} \frac{\mathrm{d}}{\mathrm{d} t} \lambda_{8}= & 2\left(\lambda_{3}^{u}\right)^{2}+2\left(\lambda_{3}^{d}\right)^{2}+6 \lambda_{6}^{2}+4 \lambda_{6} \lambda_{7}+2 \lambda_{7}^{2} \\
& +28 \lambda_{8}^{2}+\left(-16 \bar{g}_{3}^{2}+8 \bar{Y}_{q_{3 L}}^{2}\right) \lambda_{8} \\
& -4 \bar{Y}_{q_{3 L}}^{4}+\frac{13}{6} \bar{g}_{3}^{4} .
\end{aligned}
$$

Note that in all equations above we assumed real parameters. However, all formula can easily be generalized to the complex case by simply replacing a square by the absolute value squared.

By integrating these RGEs from $M$ to the stop mass scale $m_{\tilde{t}}$, we obtain the $O\left(\alpha_{3}, Y_{t, b}\right)$ contributions enhanced by $\log \left(M / m_{\tilde{t}}\right)$

\subsection{Stop masses}

In the effective theory, the stop mass matrix in the $\left(\tilde{t}_{L}, \tilde{t}_{R}\right)$ basis reads

$$
\overline{\mathcal{M}}_{\tilde{t}}^{2}=\left(\begin{array}{ll}
\bar{m}_{\tilde{Q}}^{2}+v_{u}^{2} \lambda_{1}^{u}+v_{d}^{2}\left(\lambda_{1}^{d}+\lambda_{2}^{d}\right) & v_{u} \bar{A}_{t}^{*}-v_{d} \bar{\mu}_{t}^{*} \\
v_{u} \bar{A}_{t}-v_{d} \bar{\mu}_{t} & \bar{m}_{\tilde{t}}^{2}+v_{u}^{2} \lambda_{3}^{u}+v_{d}^{2} \lambda_{3}^{d}
\end{array}\right),
$$




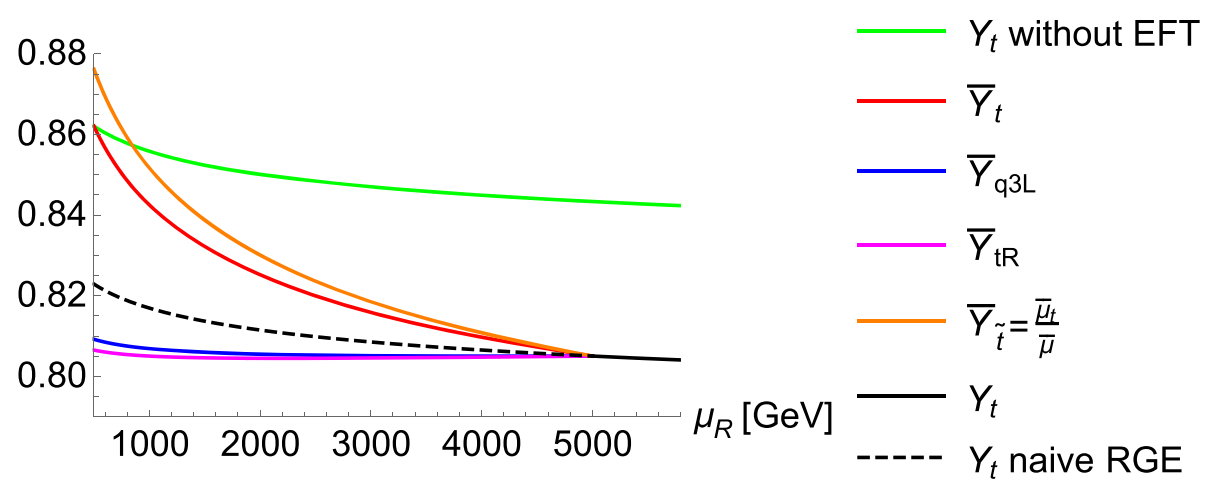

Fig. 1 Evolution of the Yukawa coupling $Y_{t}$ in the naive approach without using an EFT (green) compared to the various Higgs/Higgsinostop/top couplings in the EFT for $M=5 \mathrm{TeV}$ and $\tan \beta=50$ as a function of the renormalization scale $\mu_{R}$. Note that the only numerically sizable impact of $\tan \beta=50$ is the splitting between the $\bar{Y}_{t_{R}}$ and $\bar{Y}_{q_{3 L}}$. The initial condition of the Yukawa coupling is determined by the requirement that $v_{u} Y_{t}=m_{t}=150 \mathrm{GeV}$ at the stop scale which we choose here to be $500 \mathrm{GeV} . \bar{Y}_{\tilde{t}}=\bar{\mu}_{t} / \bar{\mu}$ shows the evolution of the $\tilde{t}-\tilde{t}-H_{d}$ coupling relative to the Higgsino mass term $\bar{\mu}$ in the EFT. We also show the projected evolution of $Y_{t}$ below the scale $M$ (blackdashed) in the MSSM RGE for the boundary condition $Y_{t}(M)=\bar{Y}_{t}(M)$. Note that above the scale $M$ SUSY is restored, so that there is only one Yukawa coupling $Y_{t}$ (black)

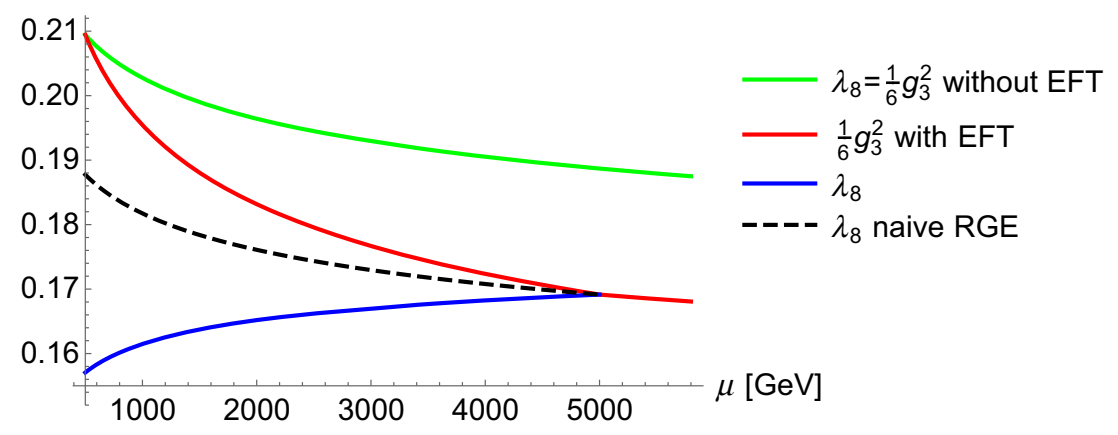

Fig. 2 Evolution of the quartic coupling to right-handed stops in the naive approach with the MSSM RGE (green) compared to the EFT approach, where $\alpha_{3}=0.1$ at the stop scale. Note that the SUSY relation $\lambda_{8}=\frac{1}{6} g_{3}^{2}$ holds only at the scale $M$ in the EFT. The dotted-black

where $v_{u, d}=\left\langle H_{u, d}^{0}\right\rangle$ are the vacuum expectation values of the Higgs scalars. By diagonalizing this matrix one obtains the stop masses and the stop mixing angle, both in the $\overline{\mathrm{MS}}$ scheme. These masses are closely related to the left-handed sbottom mass,

$M_{\tilde{b}_{L}}^{2}=\bar{m}_{\tilde{Q}}^{2}+v_{u}^{2}\left(\lambda_{1}^{u}+\lambda_{2}^{u}\right)+v_{d}^{2} \lambda_{1}^{d}$,

by $S U(2)$ gauge symmetry.

\section{Numerical analysis}

From the previous analysis, we can see that, by integrating out the gluino and the squarks of the first two generations, parameters which were originally related via SUSY in the full MSSM, do not evolve anymore in the same way in the EFT. Let us illustrate this effect with two examples where striking differences between the EFT approach and line shows the projected evolution of $\lambda_{8}$ for the boundary condition $\lambda_{8}(M)=\frac{1}{6} \bar{g}_{3}^{2}(M)$ with the naive RGE of the full MSSM. Note that above the scale $M$ SUSY is restored, $\lambda_{8}=1 / 6 g_{3}^{2}$ and evolves like $g_{3}^{2}$ in the full MSSM

the full MSSM emerge. Here we set the input parameters as $M=5 \mathrm{TeV}$, the stop mass scale $m_{\tilde{t}}=700 \mathrm{GeV}$, running top mass $m_{t}\left(m_{\tilde{t}}\right)=\bar{Y}_{t}\left(m_{\tilde{t}}\right) v_{u}=150 \mathrm{GeV}, \alpha_{3}\left(m_{\tilde{t}}\right)=0.1$, and $\tan \beta=v_{u} / v_{d}=50$. Furthermore, we have chosen the massive parameters such that the collider constraints for the Higgs mass and the stop and sbottom masses are fulfilled. This can be achieved by using the values: $\bar{m}_{\tilde{t}}\left(m_{\tilde{t}}\right)=800 \mathrm{GeV}$, $\bar{m}_{\tilde{Q}}\left(m_{\tilde{t}}\right)=900 \mathrm{GeV}, \bar{A}_{t}\left(m_{\tilde{t}}\right)=1200 \mathrm{GeV}$ which lead to a one-loop mass of $125 \mathrm{GeV}$ for the lightest Higgs, a light stop of $700 \mathrm{GeV}$ and a sbottom mass of about $900 \mathrm{GeV}$.

- The top Yukawa coupling $Y_{t}$

In the full MSSM, the Yukawa coupling $Y_{t}$ of the superpotential enters top-top-Higgs, stop-stop-Higgs couplings as well as stop-squark-Higgsino couplings in the same way. However, in the EFT these couplings are independent quantities and they evolve differently below the scale $M$. This is depicted in Fig. 1, where the evolution of $Y_{t}$ 
in the naive approach using MSSM RGE is compared to those of $\bar{Y}_{t}, \bar{Y}_{q_{3 L}}, \bar{Y}_{t_{R}}$ and $\bar{Y}_{\tilde{t}} \equiv \bar{\mu}_{t} / \bar{\mu}$ in the EFT. When the values of $\bar{Y}_{t}$ and $Y_{t}$ are determined at the stop mass scale to give the SM running top mass, their values at the scale $M$ are quite different. Note that these couplings are dimensionless and therefore do not depend on the choice of the parameters for $\bar{m}_{\tilde{t}}, \bar{m}_{\tilde{Q}}$ and $\bar{A}_{t}$.

- The quartic coupling of right-handed stops $\lambda_{8}$

In the full MSSM the quartic coupling of right-handed stops $\lambda_{8}$ is given by $\frac{1}{6} g_{3}^{2}$ by SUSY relation and evidently also evolves in the same way as $\frac{1}{6} g_{3}^{2}$. However, in the EFT $\lambda_{8}$ and $\bar{g}_{3}^{2}$ follow different RGEs below the scale $M$, as seen in Fig. 2. The relative difference at the scale $m_{\tilde{t}}$ amounts to roughly $30 \%$. Again, since $\lambda_{8}$ has no mass dimension, its running does not depend on $\bar{m}_{\tilde{t}}, \bar{m}_{\tilde{Q}}$ and $\bar{A}_{t}$.

Among the quartic scalar couplings $\lambda_{1-8}$, the running of $\lambda_{8}$ in the EFT exhibits the largest deviation from the one in the full theory. This is due to symmetry factors, leading to large coefficients of the box diagrams and self-couplings which are responsible for a change in sign on the $g_{3}^{4}$-dependence. The deviations of the other couplings $\lambda_{1-7}$ from the ones in the full theory are either positive or negative, but are smaller than $20 \%$ for our parameter set. We therefore do not show the figures of their runnings here.

\section{Conclusions}

In this article, we constructed an effective theory of the stop sector obtained from the full MSSM by integrating out the first and second generation of squarks and the gluino (which we assume to have a common mass of the order $M$ ). We computed the matching effects for the dimensionful quantities which are enhanced by powers of $M$ at $O\left(\alpha_{3}, Y_{t, b}^{2}\right)$. In addition, we obtained the complete $O\left(\alpha_{3}, Y_{t, b}^{2}\right)$ RGEs of the couplings within the EFT. In the numerical analysis we highlighted that couplings which are related via SUSY identities within the full MSSM have different RGEs within the EFT, which can lead to sizable differences. We illustrated this effect for the top Yukawa couplings and the quartic coupling of right-handed stops, finding differences up to $30 \%$ between the EFT and the naive approach. Such deviations could play a role in a future test of the stop-stop or stop-Higgs interactions which also enter the calculation of the Higgs mass.

Acknowledgements J. A. and C. G. acknowledge the support from the Swiss National Science Foundation. The work of A.C. was supported by a Marie Curie Intra-European Fellowship of the European Community's 7th Framework Programme under contract number (PIEF-GA-2012326948) and by an Ambizione Grant of the Swiss National Science Foundation.
Open Access This article is distributed under the terms of the Creative Commons Attribution 4.0 International License (http://creativecomm ons.org/licenses/by/4.0/), which permits unrestricted use, distribution, and reproduction in any medium, provided you give appropriate credit to the original author(s) and the source, provide a link to the Creative Commons license, and indicate if changes were made.

Funded by $\mathrm{SCOAP}^{3}$.

\section{Appendix}

RGEs of the full MSSM

Here we recall the RGEs of the parameters in the full MSSM, again taking into account $O\left(\alpha_{3}\right)$ and $O\left(Y_{t, b}^{2}\right)$ effects. We have

$$
\begin{aligned}
& 16 \pi^{2} \frac{\mathrm{d}}{\mathrm{d} t} g_{3}=-3 g_{3}^{3}, \\
& 16 \pi^{2} \frac{\mathrm{d}}{\mathrm{d} t} Y_{t}=Y_{t}\left[-\frac{16}{3} g_{3}^{2}+6 Y_{t}^{2}+Y_{b}^{2}\right] \text {, } \\
& 16 \pi^{2} \frac{\mathrm{d}}{\mathrm{d} t} Y_{b}=Y_{b}\left[-\frac{16}{3} g_{3}^{2}+Y_{t}^{2}+6 Y_{b}^{2}\right] \text {, } \\
& 16 \pi^{2} \frac{\mathrm{d}}{\mathrm{d} t} m_{H_{u}}^{2}=6\left(Y_{t}^{2}\left(m_{H_{u}}^{2}+m_{\tilde{Q}}^{2}+m_{\tilde{t}}^{2}\right)+A_{t}^{2}\right), \\
& 16 \pi^{2} \frac{\mathrm{d}}{\mathrm{d} t} m_{H_{d}}^{2}=6\left(Y_{b}^{2}\left(m_{H_{d}}^{2}+m_{\tilde{Q}}^{2}+m_{\tilde{b}_{R}}^{2}\right)+A_{b}^{2}\right), \\
& 16 \pi^{2} \frac{\mathrm{d}}{\mathrm{d} t} m_{H_{d} H_{u}}^{2}=3\left(Y_{t}^{2}+Y_{b}^{2}\right) m_{H_{d} H_{u}}^{2}+6\left(Y_{t} A_{t}+Y_{b} A_{b}\right) \mu, \\
& 16 \pi^{2} \frac{\mathrm{d}}{\mathrm{d} t} m_{\tilde{Q}}^{2}=-\frac{32}{3} g_{3}^{2} m_{\tilde{g}}^{2}+2 Y_{t}^{2}\left(m_{\tilde{Q}}^{2}+m_{H_{u}}^{2}+m_{\tilde{t}}^{2}\right) \\
& +2 Y_{b}^{2}\left(m_{\tilde{Q}}^{2}+m_{H_{d}}^{2}+m_{\tilde{b}_{R}}^{2}\right)+2\left(A_{t}^{2}+A_{b}^{2}\right) \text {, } \\
& 16 \pi^{2} \frac{\mathrm{d}}{\mathrm{d} t} m_{\tilde{t}}^{2}=-\frac{32}{3} g_{3}^{2} m_{\tilde{g}}^{2}+4 Y_{t}^{2}\left(m_{\tilde{Q}}^{2}+m_{\tilde{t}}^{2}+m_{H_{u}}^{2}\right) \\
& +4 A_{t}^{2} \text {, } \\
& 16 \pi^{2} \frac{\mathrm{d}}{\mathrm{d} t} m_{\tilde{b}_{R}}^{2}=-\frac{32}{3} g_{3}^{2} m_{\tilde{g}}^{2}+4 Y_{b}^{2}\left(m_{\tilde{Q}}^{2}+m_{\tilde{b}}^{2}+m_{H_{d}}^{2}\right) \\
& +4 A_{b}^{2} \text {, } \\
& 16 \pi^{2} \frac{\mathrm{d}}{\mathrm{d} t} \mu=3\left(Y_{t}^{2}+Y_{b}^{2}\right) \mu \text {, } \\
& 16 \pi^{2} \frac{\mathrm{d}}{\mathrm{d} t} A_{t}=A_{t}\left[-\frac{16}{3} g_{3}^{2}+18 Y_{t}^{2}+Y_{b}^{2}\right] \\
& +2 Y_{t} Y_{b} A_{b}+\frac{32}{3} g_{3}^{2} m_{\tilde{g}} Y_{t} \text {, } \\
& 16 \pi^{2} \frac{\mathrm{d}}{\mathrm{d} t} A_{b}=A_{b}\left[-\frac{16}{3} g_{3}^{2}+Y_{t}^{2}+18 Y_{b}^{2}\right] \\
& +2 Y_{t} Y_{b} A_{t}+\frac{32}{3} g_{3}^{2} m_{\tilde{g}} Y_{b}, \\
& 16 \pi^{2} \frac{\mathrm{d}}{\mathrm{d} t} m_{\tilde{g}}=-6 g_{3}^{2} m_{\tilde{g}} \text {. }
\end{aligned}
$$

\section{References}

1. S. Dimopoulos, G.F. Giudice, Phys. Lett. B 357, 573 (1995). https:// doi.org/10.1016/0370-2693(95)00961-J. arXiv:hep-ph/9507282

2. G.F. Giudice, A. Romanino, Nucl. Phys. B 699, 65 (2004). https://doi.org/10.1016/j.nuclphysb.2004.11.048. https://doi.org/ 
10.1016/j.nuclphysb.2004.08.001. arXiv:hep-ph/0406088 [Erratum: Nucl. Phys. B 706, 487 (2005)]

3. K. Inoue, A. Kakuto, H. Komatsu, S. Takeshita, Prog. Theor. Phys. 68, 927 (1982). https://doi.org/10.1143/PTP.68.927 [Erratum: Prog. Theor. Phys. 70, 330 (1983)]

4. K. Inoue, A. Kakuto, H. Komatsu, S. Takeshita, Prog. Theor. Phys. 71, 413 (1984). https://doi.org/10.1143/PTP.71.413

5. J.P. Derendinger, C.A. Savoy, Nucl. Phys. B 237, 307 (1984). https://doi.org/10.1016/0550-3213(84)90162-7

6. B. Gato, J. Leon, J. Perez-Mercader, M. Quiros, Nucl. Phys. B 253, 285 (1985). https://doi.org/10.1016/0550-3213(85)90532-2

7. N.K. Falck, Z. Phys. C 30, 247 (1986). https://doi.org/10.1007/ BF01575432

8. S.P. Martin, M.T. Vaughn, Phys. Rev. D 50, 2282 (1994) https://doi. org/10.1103/PhysRevD.50.2282. 10.1103/PhysRevD.78.039903. arXiv:hep-ph/9311340 [Erratum: Phys. Rev. D 78, 039903 (2008)]

9. G. Aad et al., ATLAS Collaboration, Phys. Lett. B 716, 1 (2012). https://doi.org/10.1016/j.physletb.2012.08.020. arXiv:1207.7214 [hep-ex]

10. S. Chatrchyan et al., CMS Collaboration, Phys. Lett. B 716, 30 (2012). https://doi.org/10.1016/j.physletb.2012.08.021. arXiv:1207.7235 [hep-ex]

11. J.R. Ellis, G. Ridolfi, F. Zwirner, Phys. Lett. B 257, 83 (1991). https://doi.org/10.1016/0370-2693(91)90863-L

12. Y. Okada, M. Yamaguchi, T. Yanagida, Prog. Theor. Phys. 85, 1 (1991). https://doi.org/10.1143/PTP.85.1

13. H.E. Haber, R. Hempfling, Phys. Rev. Lett. 66, 1815 (1991). https:// doi.org/10.1103/PhysRevLett.66.1815

14. U. Ellwanger, C. Hugonie, A.M. Teixeira, Phys. Rep. 496, 1 (2010). https://doi.org/10.1016/j.physrep.2010.07.001. arXiv:0910.1785 [hep-ph]

15. L.J. Hall, D. Pinner, J.T. Ruderman, JHEP 1204, 131 (2012). https:// doi.org/10.1007/JHEP04(2012)131. arXiv:1112.2703 [hep-ph]

16. M. Chala, A. Delgado, G. Nardini, M. Quiros, JHEP 1704, 097 (2017). https://doi.org/10.1007/JHEP04(2017)097. arXiv:1702.07359 [hep-ph]

17. P. Batra, A. Delgado, D.E. Kaplan, T.M.P. Tait, JHEP 0402, 043 (2004). https://doi.org/10.1088/1126-6708/2004/02/ 043. arXiv:hep-ph/0309149

18. J.R. Espinosa, M. Quiros, Phys. Rev. Lett. 81, 516 (1998). https:// doi.org/10.1103/PhysRevLett.81.516. arXiv:hep-ph/9804235

19. A. Djouadi, Phys. Rep. 459, 1 (2008). https://doi.org/10.1016/j. physrep.2007.10.005. arXiv:hep-ph/0503173

20. F. Brummer, S. Kraml, S. Kulkarni, JHEP 1208, 089 (2012). https:// doi.org/10.1007/JHEP08(2012)089. arXiv:1204.5977 [hep-ph]

21. C. Wymant, Phys. Rev. D 86, 115023 (2012). https://doi.org/10. 1103/PhysRevD.86.115023. arXiv:1208.1737 [hep-ph]

22. G. Aad et al., ATLAS Collaboration, JHEP 1406, 124 (2014). https://doi.org/10.1007/JHEP06(2014)124. arXiv:1403.4853 [hep-ex]

23. The ATLAS collaboration [ATLAS Collaboration], ATLASCONF-2016-050

24. S. Chatrchyan et al., CMS Collaboration, Eur. Phys. J. C 73(12), 2677 (2013) https://doi.org/10.1140/epjc/s10052-013-2677-2. arXiv:1308.1586 [hep-ex]

25. H.C. Cheng, C. Gao, L. Li, N.A. Neill, JHEP 1605, 036 (2016). https://doi.org/10.1007/JHEP05(2016)036. arXiv:1604.00007 [hep-ph]

26. W. Porod, T. Wohrmann, Phys. Rev. D 55, 2907 (1997). https://doi. org/10.1103/PhysRevD.67.059902. 10.1103/PhysRevD.55.2907. arXiv:hep-ph/9608472 [Erratum: Phys. Rev. D 67, 059902 (2003)]

27. The ATLAS collaboration [ATLAS Collaboration], ATLASCONF-2016-076

28. M. Aaboud et al., ATLAS Collaboration, Phys. Rev. D 94(3), 032005 (2016). https://doi.org/10.1103/PhysRevD.94.032005. arXiv:1604.07773 [hep-ex]
29. J. Aebischer, A. Crivellin, C. Greub, Phys. Rev. D 91(3), 035010 (2015). https://doi.org/10.1103/PhysRevD.91.035010. arXiv:1410.8459 [hep-ph]

30. R. Grber, M.M. Mhlleitner, E. Popenda, A. Wlotzka, Eur. Phys. J. C 75, 420 (2015). https://doi.org/10.1140/epjc/s10052-015-3626-Z. arXiv: 1408.4662 [hep-ph]

31. G. Aad et al., ATLAS Collaboration, Phys. Rev. D 90(5), 052008 (2014). https://doi.org/10.1103/PhysRevD.90.052008. arXiv: 1407.0608 [hep-ex]

32. S. Macaluso, M. Park, D. Shih, B. Tweedie, JHEP 1603, 151 (2016). https://doi.org/10.1007/JHEP03(2016)151. arXiv:1506.07885 [hep-ph]

33. A. Belyaev, V. Sanz, M. Thomas, JHEP 1601, 102 (2016). https:// doi.org/10.1007/JHEP01(2016)102. arXiv:1510.07688 [hep-ph]

34. A. Kobakhidze, N. Liu, L. Wu, J.M. Yang, M. Zhang, Phys. Lett. B 755, 76 (2016). https://doi.org/10.1016/j.physletb.2016.02.003. arXiv:1511.02371 [hep-ph]

35. A. Crivellin, U. Haisch, L.C. Tunstall, JHEP 1609, 080 (2016). https://doi.org/10.1007/JHEP09(2016)080. arXiv:1604.00440 [hep-ph]

36. The ATLAS collaboration [ATLAS Collaboration], ATLASCONF-2016-054

37. T. Sakuma, CMS Collaboration, PoS LHCP 2016, 145 (2017). arXiv:1609.07445 [hep-ex]

38. The ATLAS collaboration [ATLAS Collaboration], ATLASCONF-2017-038

39. A.M. Sirunyan et al., CMS Collaboration, arXiv:1707.07274 [hepex]

40. G. Aad et al., ATLAS Collaboration, Eur. Phys. J. C 75(5), 208 (2015). https://doi.org/10.1140/epjc/s10052-015-3408-7. arXiv: 1501.07110 [hep-ex]

41. The ATLAS collaboration [ATLAS Collaboration], ATLASCONF-2016-096

42. C. Arina, M. Chala, V. Martin-Lozano, G. Nardini, JHEP 1612, 149 (2016). https://doi.org/10.1007/JHEP12(2016)149. arXiv:1610.03822 [hep-ph]

43. V. Khachatryan et al., CMS Collaboration, Phys. Rev. D 90(9), 092007 (2014). https://doi.org/10.1103/PhysRevD.90. 092007. arXiv:1409.3168 [hep-ex]

44. CMS Collaboration [CMS Collaboration], CMS-PAS-SUS-16-024

45. G. Aad et al., ATLAS Collaboration, JHEP 1405, 071 (2014), https://doi.org/10.1007/JHEP05(2014)071. arXiv:1403.5294 [hep-ex]

46. A. Bharucha, S. Heinemeyer, F. von der Pahlen, Eur. Phys. J. C 73(11), 2629 (2013). https://doi.org/10.1140/epjc/ s10052-013-2629-x. arXiv:1307.4237 [hep-ph]

47. T.A.W. Martin, D. Morrissey, JHEP 1412, 168 (2014). https://doi. org/10.1007/JHEP12(2014)168. arXiv:1409.6322 [hep-ph]

48. L. Calibbi, J.M. Lindert, T. Ota, Y. Takanishi, JHEP 1411, 106 (2014). https://doi.org/10.1007/JHEP11(2014)106. arXiv: 1410.5730 [hep-ph]

49. M. Chakraborti, U. Chattopadhyay, A. Choudhury, A. Datta, S. Poddar, JHEP 1511, 050 (2015). https://doi.org/10.1007/ JHEP11(2015)050. arXiv:1507.01395 [hep-ph]

50. The ATLAS collaboration [ATLAS Collaboration], ATLASCONF-2014-010

51. V. Khachatryan et al., CMS Collaboration, Phys. Rev. D 90, 112013 (2014). https://doi.org/10.1103/PhysRevD.90.112013. arXiv: 1410.2751 [hep-ex]

52. A. Celis, V. Ilisie, A. Pich, JHEP 1307, 053 (2013). https://doi.org/ 10.1007/JHEP07(2013)053. arXiv:1302.4022 [hep-ph]

53. C.W. Chiang, K. Yagyu, JHEP 1307, 160 (2013). https://doi.org/ 10.1007/JHEP07(2013)160. arXiv:1303.0168 [hep-ph]

54. C.Y. Chen, S. Dawson, M. Sher, Phys. Rev. D 88, 015018 (2013). https://doi.org/10.1103/PhysRevD.88.015018. 10.1103/ 
PhysRevD.88.039901. arXiv:1305.1624 [hep-ph] [Erratum: Phys. Rev. D 88, 039901 (2013)]

55. N. Craig, J. Galloway, S. Thomas, arXiv:1305.2424 [hep-ph]

56. L. Wang, X.F. Han, JHEP 1411, 085 (2014). https://doi.org/10. 1007/JHEP11(2014)085. arXiv:1404.7437 [hep-ph]

57. B. Grinstein, P. Uttayarat, JHEP 1306, 094 (2013). https:// doi.org/10.1007/JHEP09(2013)110. 10.1007/JHEP06(2013)094. arXiv:1304.0028 [hep-ph] [Erratum: JHEP 1309, 110 (2013)]

58. L. Wang, F. Zhang, X.F. Han, Phys. Rev. D 95(11), 115014 (2017). https://doi.org/10.1103/PhysRevD.95.115014. arXiv:1701.02678 [hep-ph]

59. M. Aaboud et al., ATLAS Collaboration, Eur. Phys. J. C 76(11), 585 (2016). https://doi.org/10.1140/epjc/s10052-016-4400-6. arXiv:1608.00890 [hep-ex]

60. V. Khachatryan et al., CMS Collaboration, JHEP 1410, 160 (2014). https://doi.org/10.1007/JHEP10(2014)160. arXiv:1408.3316 [hep-ex]

61. N. Arkani-Hamed, S. Dimopoulos, G.F. Giudice, A. Romanino, Nucl. Phys. B 709, 3 (2005). https://doi.org/10.1016/j.nuclphysb. 2004.12.026. arXiv:hep-ph/0409232

62. M. Muhlleitner, H. Rzehak, M. Spira, JHEP 0904, 023 (2009). https://doi.org/10.1088/1126-6708/2009/04/023. arXiv:0812.3815 [hep-ph]

63. M. Carena, G. Nardini, M. Quiros, C.E.M. Wagner, JHEP 0810, 062 (2008). https://doi.org/10.1088/1126-6708/2008/10/ 062. arXiv:0806.4297 [hep-ph]

64. G.F. Giudice, A. Strumia, Nucl. Phys. B 858, 63 (2012). https://doi. org/10.1016/j.nuclphysb.2012.01.001. arXiv:1108.6077 [hep-ph]

65. A. Pomarol, M. Quiros, Phys. Lett. B 438, 255 (1998). https://doi. org/10.1016/S0370-2693(98)00979-4. arXiv:hep-ph/9806263

66. S. Dimopoulos, K. Howe, J. March-Russell, Phys. Rev. Lett. 113, 111802 (2014). https://doi.org/10.1103/PhysRevLett.113.111802. arXiv:1404.7554 [hep-ph]

67. I. Garcia Garcia, K. Howe, J. March-Russell, JHEP 1512, 005 (2015). https://doi.org/10.1007/JHEP12(2015)005. arXiv:1510.07045 [hep-ph]

68. A. Delgado, M. Garcia-Pepin, G. Nardini, M. Quiros, Phys. Rev. D 94(9), 095017 (2016). https://doi.org/10.1103/PhysRevD.94. 095017. arXiv:1608.06470 [hep-ph]

69. P.H. Chankowski, Phys. Rev. D 41, 2877 (1990). https://doi.org/ 10.1103/PhysRevD.41.2877

70. K.i. Hikasa, Y. Nakamura, Z. Phys. C 70, 139 (1996). https://doi.org/10.1007/BF02906995. 10.1007/s002880050091. arXiv:hep-ph/9501382 [Erratum: Z. Phys. C 71, 356 (1996)]

71. M.M. Nojiri, K. Fujii, T. Tsukamoto, Phys. Rev. D 54, 6756 (1996). https://doi.org/10.1103/PhysRevD.54.6756. arXiv:hep-ph/9606370

72. H.C. Cheng, J.L. Feng, N. Polonsky, Phys. Rev. D 56, 6875 (1997). https://doi.org/10.1103/PhysRevD.56.6875. arXiv:hep-ph/9706438
73. H.C. Cheng, J.L. Feng, N. Polonsky, Phys. Rev. D 57, 152 (1998). https://doi.org/10.1103/PhysRevD.57.152. arXiv:hep-ph/9706476

74. M.M. Nojiri, D.M. Pierce, Y. Yamada, Phys. Rev. D 57, 1539 (1998). https://doi.org/10.1103/PhysRevD.57.1539. arXiv:hep-ph/9707244

75. E. Katz, L. Randall, Sf Su, Nucl. Phys. B 536, 3 (1998). https:// doi.org/10.1016/S0550-3213(98)00632-4. arXiv:hep-ph/9801416

76. S. Kiyoura, M.M. Nojiri, D.M. Pierce, Y. Yamada, Phys. Rev. D 58, 075002 (1998). https://doi.org/10.1103/PhysRevD.58.075002. arXiv:hep-ph/9803210

77. R. Hempfling, Phys. Rev. D 49, 6168 (1994). https://doi.org/10. 1103/PhysRevD.49.6168

78. L.J. Hall, R. Rattazzi, U. Sarid, Phys. Rev. D 50, 7048 (1994). https://doi.org/10.1103/PhysRevD.50.7048. arXiv:hep-ph/9306309

79. M. Carena, M. Olechowski, S. Pokorski, C.E.M. Wagner, Nucl. Phys. B 426, 269 (1994). https://doi.org/10.1016/ 0550-3213(94)90313-1. arXiv:hep-ph/9402253

80. D. Noth, M. Spira, Phys. Rev. Lett. 101, 181801 (2008). https://doi org/10.1103/PhysRevLett.101.181801. arXiv:0808.0087 [hep-ph]

81. L. Hofer, U. Nierste, D. Scherer, JHEP 0910, 081 (2009). https:// doi.org/10.1088/1126-6708/2009/10/081. arXiv:0907.5408 [hep$\mathrm{ph}]$

82. A. Crivellin, Phys. Rev. D 83, 056001 (2011). https://doi.org/10 1103/PhysRevD.83.056001. arXiv:1012.4840 [hep-ph]

83. A. Crivellin, L. Hofer, J. Rosiek, JHEP 1107, 017 (2011). https:// doi.org/10.1007/JHEP07(2011)017. arXiv:1103.4272 [hep-ph]

84. A. Crivellin, C. Greub, Phys. Rev. D 87, 015013 (2013). https:// doi.org/10.1103/PhysRevD.87.015013. 10.1103/PhysRevD.87. 079901. arXiv:1210.7453 [hep-ph] [Erratum: Phys. Rev. D 87, 079901 (2013)]

85. P. Fayet, S. Ferrara, Phys. Rep. 32, 249 (1977). https://doi.org/10. 1016/0370-1573(77)90066-7

86. P. Fayet, Phys. Lett. 64B, 159 (1976). https://doi.org/10.1016/ 0370-2693(76)90319-1

87. H.E. Haber, G.L. Kane, Phys. Rep. 117, 75 (1985). https://doi.org/ 10.1016/0370-1573(85)90051-1

88. J. Rosiek, arXiv:hep-ph/9511250

89. P.C. West, Phys. Lett. 137B, 371 (1984). https://doi.org/10.1016/ 0370-2693(84)91734-9

90. D.R.T. Jones, L. Mezincescu, Phys. Lett. 138B, 293 (1984). https:// doi.org/10.1016/0370-2693(84)91663-0

91. S.P. Martin, M.T. Vaughn, Phys. Lett. B 318, 331 (1993). https:// doi.org/10.1016/0370-2693(93)90136-6. arXiv:hep-ph/9308222

92. Y. Yamada, Phys. Rev. Lett. 72, 25 (1994). https://doi.org/10.1103/ PhysRevLett.72.25. arXiv:hep-ph/9308304 